
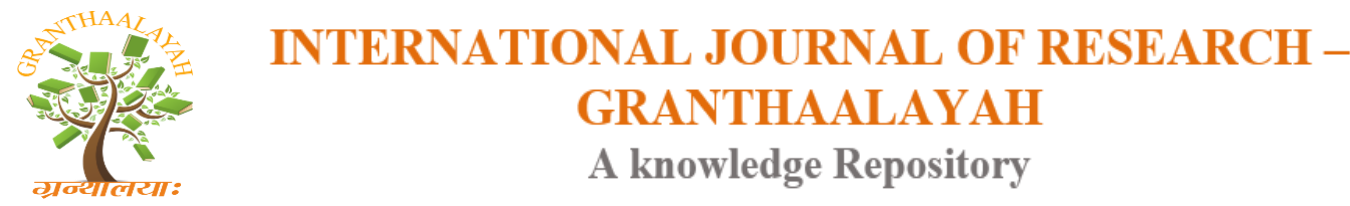

Science

\title{
STUDY OF SOLID STATE PHYSICS AND FABRICATION OF NANOSTRUCTURES
}

\author{
Dr. S. K. Mahobia ${ }^{1}$, Prof. G.R.Kumrey ${ }^{2}$ \\ ${ }^{1}$ Assistant Professor, Department of Physics, Rewa Engineering College, Rewa (M.P.), INDIA \\ ${ }^{2}$ Assistant Professor, Department of Electrical Engg., Rewa Engineering College, Rewa (M.P.), \\ INDIA
}

DOI: https://doi.org/10.29121/granthaalayah.v4.i8.2016.2571

\section{ABSTRACT}

These factors play roles to various degrees (but not 100\%) of importance. For example, the confinement and the coherent effects are not as complete as that in an atom. Both the crystalline (bulk) states and the surface/interface states cannot be ignored in nanoscale structures. The different mixture of atomic/molecular, mesoscopic and macroscopic characters make the properties of nanostructures vary dramatically. Nanostructural materials are often in a metastable state. Their detailed atomic configuration depends sensitively on the kinetic processes in which they are fabricated. Therefore, the properties of nanostructures can be widely adjustable by changing their size, shape and processing conditions.

Keywords:

nanostructures, crystalline, semiconductor layer.

Cite This Article: Dr. S. K. Mahobia, and Prof. G.R.Kumrey, "STUDY OF SOLID STATE PHYSICS AND FABRICATION OF NANOSTRUCTURES" International Journal of Research - Granthaalayah, Vol. 4, No. 8 (2016): 136-140.

\section{INTRODUCTION}

The word "nano" means a billionth $\left(10^{-9}\right)$ part of a unit in general. In our course, it refers specifically to the length scale: nanometer or $\mathrm{nm}$,

$$
1 \mathrm{~nm}=10^{-9} \mathrm{~m}=10^{-3} \mu \mathrm{m}=10 \AA
$$

Nanostructures refer to materials systems with length scale in the range of $\sim 1-100 \mathrm{~nm}$ in at least one dimension. In a nanostructure, electrons are confined in the nanoscale dimension(s), but are free to move in other dimension(s). One way to classify nanostructures is based on the dimensions in which electrons move freely: 
Quantum well: electrons are confined in one dimension (1D), free in other 2D. It can be realized by sandwiching a narrow-bandgap semiconductor layer between the wide-gap ones. A quantum well is often called a $2 \mathrm{D}$ electronic system.

Quantum wires: confined in two dimensions, free in 1D (so it is called a 1D electronic system). Real quantum wires include polymer chains, nanowires and nanotubes.

Quantum dots: electrons are confined in all dimensions, as in clusters and nano-crystallites Certainly, we know that the properties are changed greatly when the wire is divided into individual $\mathrm{Cu}$ atoms (even more at the level of electrons, protons and neutrons). Significant property changes often start when we get down to the nanoscales. The following phenomena critically affect the properties of nanostructural materials.

Quantum confinement: the confinement of electrons in the nanoscale dimensions result in quantization of energy and momentum, and reduced dimensionality of electronic states.

Quantum coherence: certain phase relation of wave function is preserved for electrons moving in a nanostructure, so wave interference effect must be considered. But in nanostructures, generally the quantum coherence is not maintained perfectly as in atoms and molecules. The coherence is often disrupted to some extent by defects in the nanostructures. Therefore, both quantum coherent and de-coherent effects have to be considered, which often makes the description of electronic motion in a nanostructure more complicated than in the extreme cases.

Surface/interface effects: a significant fraction (even the majority) of atoms in nanostructure is located at and near the surfaces or interfaces. The mechanic, thermodynamic, electronic, magnetic, optical and chemical states of these atoms can be quite different than those interior atoms. The situation is similar to molecular behavior in chemistry (e.g., $\mathrm{N}$ vs. $\mathrm{N}_{2}$ ) in certain aspect. Because of the rich and often surprising outcomes, it will be extremely interesting and challenging to play with nanostructural systems. Nanoscience and nano-engineering have been an area where many breakthroughs have been and will continue to be produced.

\section{ATOMIC STRUCTURE}

In examining atomic structure though, we have to clarify this statement. An atom cannot be broken down further without changing the chemical nature of the substance. For example, if you have 1 kilogram, 1 gram or 1 atom of nitrogen, all of these units have the same properties. We can break down the atom of nitrogen into smaller particles; however, when we do, the atom loses its chemical properties. Here we learn that: that each atom is made up of a combination of three types of particles: electrons, protons, and neutrons.

Use the references including multimedia resources and

- Prepare short notes detailing the different properties of these three particles.

- Describe how the existence of molecules/atoms can be demonstrated by Brownian motion experiment. Use the references provided to do this. 


\section{AVOGADRO'S CONSTANT, THE MOLE}

Make a short note on atomic and molecular masses and describe how they are measured. Emphasize that on the carbon scale, the atomic mass of carbon-12 is taken as exactly 12, making that of hydrogen 1.008 and that of oxygen 16.

\section{ATOMIC BONDS}

In covalent bonding, electron sharing occurs between two or more atoms. For example, each carbon atom (C), Fig. 1.1 has four outer electrons, and all can be shared with four other carbon atoms to make four bonds, each consisting of two interlocking electron clouds.

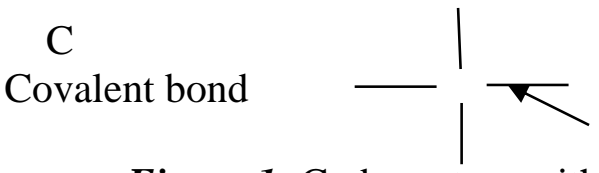

Figure 1: Carbon atom with its four bonds

\section{CRYSTAL}

In crystallography a crystal structure is a unique arrangement of atoms in a crystal. A crystal structure is composed of a unit cell, a set of atoms arranged in a particular way; which is periodically repeated in three dimensions on a lattice. The spacing between unit cells in various directions is called its lattice parameters. The symmetry properties of the crystal are embodied in its space group. A crystal's structure and symmetry play a role in determining many of its properties, such as cleavage, electronic band structure, and optical properties.

1) In order to understand some of these concepts we must know for example the meaning of the lattice

2) A lattice Is a simplified geometry of a crystal in which the atoms are swept away leaving only a skeleton of mathematical points whereby each point replaces anything from one to several hundred of original atoms. Each group of these atoms is called the basis

3) In two dimensions, there are only five lattices: square, rectangular, centred rectangular, oblique and hexagonal. These are generally known as Bravias lattice.

\section{CLASSIFICATION OF CRYSTALS BY SYMMETRY}

The defining property of a crystal is its inherent symmetry, by which we mean that under certain operations the crystal remains unchanged when viewed from a given direction. For example, rotating the crystal 180 degrees about a certain axis may result in an atomic configuration which is identical to the original configuration. The crystal is then said to have a two-fold rotational symmetry about this axis. In addition to rotational symmetries like this, a crystal may have symmetries in the form of mirror planes and translational symmetries, and also the so-called compound symmetries which are a combination of translation and rotation/mirror symmetries.

1) Read about this and solve as many problems as possible. Use the references provided. Determine the number of rotational symmetry for rectangular, circular, equilateral, and hexagonal lattices. 
2) When a square lattice shown in Fig. is rotated about an axis through it centre, it will appear exactly the same four times after being rotated through $360^{\circ}$. Thus, a square lattice has a four-fold rotational symmetry. In each fold, the lattice is rotated through $90^{\circ}$ or $\pi / 2$

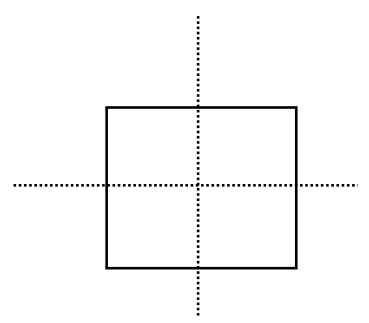

Figure 2: A square lattice

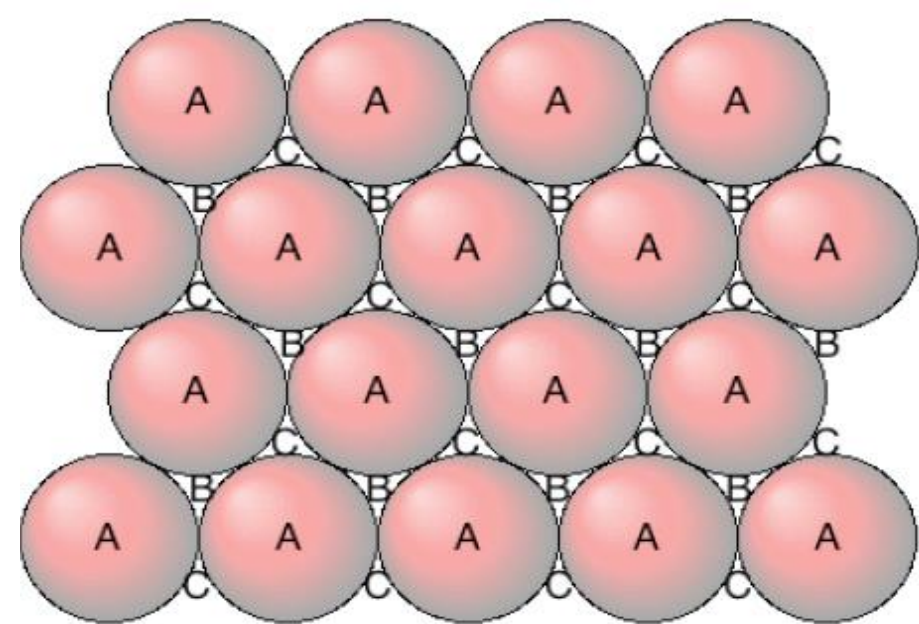

Figure 3: Stacking sequence in a simple closed-packed structure

Spheres are arranged in a single closest-packed layer A by placing each sphere in contact with six others. This layer may serve as either the basal plane of an hcp structure or the (111) plane of the fcc structure. A second and identical layer of spheres can be placed on top of this, above and parallel to the plane of the drawing with centres over the points marked B. There are two choices for a third layer. It can go in over $\mathrm{A}$ or over $\mathrm{C}$. If it goes in over $\mathrm{A}$ the sequence is $\mathrm{ABABAB} . .$. and the structure is hexagonal close-packed. If the third layer goes in over $\mathrm{C}$ the

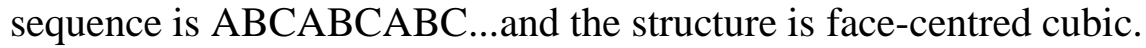

\section{PACKING FRACTION}

The lattice point in this case refers to a complete number of atoms that a unit cell contains. In a sc, the atoms are in contact with one another only along the edges of the cell as shown in Fig. 


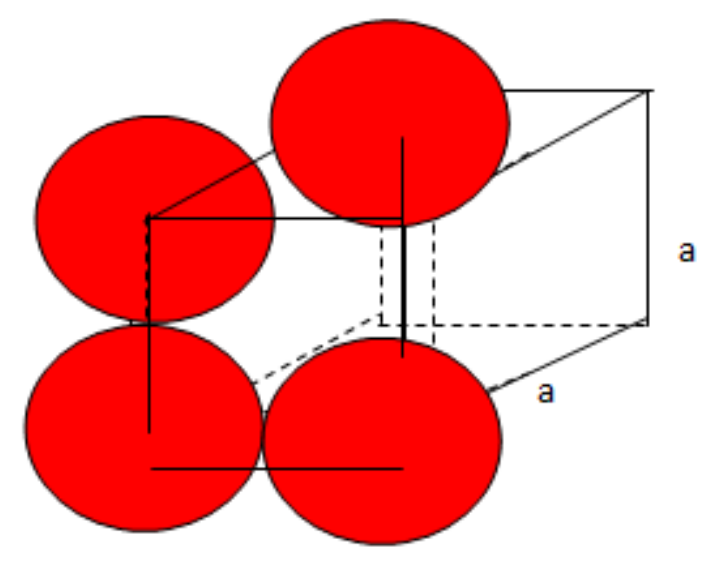

Figure 4: A simple cubic lattice showing relative positions of atoms on one face.

\section{REFERENCES}

[1] The Gamma Cell 220 Excel Co 60 Irradiation Facility, "Nordion, Canada", catalog, 2002.

[2] Agilent Gillent 4155B/4156B "Simeconductor Parameter Analyzer " User's Guide Measurement and analysis Agilent part No. 04156-90200 catalog, 2000.

[3] Ugur Cilingiroglu, "Systematic Analysis of Bipolar and MOS Transistor", Artech House Inc., 1993.

[4] Electric Circuits" by James Nelson (Pearson publication)

[5] Basic Electrical Engg." By DC Kulshreshtha, TMHill.

[6] Malcom Goodge, "Semiconductor Device Technology'", prentice - Hall, Inc., 1983.

[7] Tomas L. Floyd, "Electreonic Devices", prentice - Hall, Inc., 1996.

[8] Henry B.Weisbecker, "Solid State Devices and Integrated Circuits ", Reston and prenticHall Company Inc., 1982.

[9] Neil Storey, " Electronics Systems Approach ", Addison Wesley publishing company Inc., 1992.

[10] E.Simoen, " Radiation Effects in Advanced Semiconductor Materials and Devices ", Springer-Verlay Berlin Heidelberg Inc., 2002 